\title{
Unchanged gastric emptying and visceral perception in early Parkinson's disease after a high caloric test meal
}

\author{
L. Epprecht ${ }^{1}$ - S. R. Schreglmann ${ }^{2,6}$ - O. Goetze G,5 $^{3,5}$ Doitalla ${ }^{4}$ C. R. Baumann ${ }^{2}$. \\ D. Waldvogel ${ }^{2}$
}

Received: 30 March 2015/Revised: 23 May 2015/Accepted: 25 May 2015/Published online: 6 June 2015

(C) Springer-Verlag Berlin Heidelberg 2015

\begin{abstract}
Delayed gastric emptying (GE) is a frequent non-motor feature in Parkinsońs disease (PD). This prospective study (clinicaltrials.gov Identifier NCT01518751) investigated GE and visceral perception in early motor phase PD patients in comparison to age-matched and younger controls. In addition, the effect of Levodopa on GE was assessed in healthy aged controls. 16 PD patients (Hoehn \& Yahr 2), 11 sex-/age-matched Ctrl1 and 10 young, male Ctrl2 subjects were subjected to a high caloric $(428 \mathrm{kcal}){ }^{13} \mathrm{C}$-Sodium Octanoate breath test strictly OFF dopaminergic medication. Visceral appetite sensation was monitored using visual analogue scales (VAS). GE was similarly studied in 7 controls ON/OFF oral Levodopa. GE was not altered in PD patients compared to age-/sex-matched and younger controls $(p=0.76)$. Subjective appetite perception was not altered in the PD group in comparison to Ctrl1, but was
\end{abstract}

L. Epprecht, S. R. Schreglmann contributed equally.

$\triangle$ S. R. Schreglmann

sebastian.schreglmann@gmail.com

1 Department of Neurosurgery, Kantonsspital St.Gallen, St.Gallen, Switzerland

2 Department of Neurology, University Hospital Zurich, Zurich, Switzerland

3 Division of Gastroenterology, University Hospital Zurich, Zurich, Switzerland

4 Department of Neurology, St. Josef-Hospital, Ruhr-University of Bochum, Bochum, Germany

5 Division of Hepatology, Department of Medicine II, University Hospital Wuerzburg, Würzburg, Germany

6 Present Address: Department of Neurology, Kantonsspital St.Gallen, Rorschacher Strasse 95, 9007 St.Gallen, Switzerland significantly higher in Ctrl2 subjects $(p=0.02) .100 \mathrm{mg}$ oral Levodopa/25 mg Benserazide significantly slowed GE by $18 \%$ among healthy controls $(p=0.04)$. In early motor stage PD OFF dopaminergic medication, there was no GE slowing after a high caloric test meal. Levodopa, however, caused a robust GE slowing in healthy aged individuals. Our data indicate that clinically relevant GE slowing in early PD is related to the iatrogenic effect of dopamine treatment. Subjective appetite perception is not affected in this disease stage. This data add to the understanding of gastrointestinal symptoms in early motor stage PD and highlight the influence of dopaminergic medication.

Keywords Gastric emptying · Parkinson's disease · High caloric test meal - Appetite sensation - Dopaminergic treatment

\section{Introduction}

Due to the increasing recognition of its non-motor features, Parkinson's disease (PD) is nowadays recognized as a systemic alpha-synucleinopathy rather than an isolated disease of basal ganglia motor control [1]. Symptoms of autonomic dysfunction are among the most disturbing nonmotor features in PD [2] and their prevalence seems to increase with advanced disease stages [3]. Several studies show that gastrointestinal (GI) symptoms account for much of the autonomic dysfunction disease burden [4, 5]. Delayed gastric emptying (GE) refers to a slowing in gastric motility in the absence of mechanical obstruction and has repeatedly been reported in PD patients of different disease stages [6-12]. However, some studies measured GE under the influence of dopaminergic medication [6, 7], some used controls of a different age [6], some used liquid 
or semi-liquid test meals [12], some included patients in advanced PD disease stages only [10] or assessed GE only with a low-caloric (240 kcal-calorically equalling one butter croissant) test meal $[7,8,11]$. The question therefore remains whether there is altered GE after a medium size meal in early disease stages in the absence of potentially interfering medication. We investigated this using a ${ }^{13} \mathrm{C}$ Sodium Octanoate breath test (OBT) paradigm strictly OFF dopaminergic medication using a $428 \mathrm{kcal}$ meal, more closely representing a standard meal with regards to caloric and nutritional content.

A considerable number of PD patients display unintended weight loss during the course of their disease and this can be dramatic in some [13]. It is known that low initial body weight and weight loss during the course of PD is associated with increased risk of dyskinesia, malnutrition and frailty [14], while loss of appetite, constipation and early satiety have the highest influence on food intake in malnourished PD patients [15]. We therefore assessed visceral perception for appetite during ingestion in the same group of early motor phase PD patients and control subjects using repetitive visual analogue scales (VAS).

In addition to disease-inherent mechanisms, GI motility is also subjected to iatrogenic influence in PD: it has been shown previously using radioscintigraphy experiments that dopaminergic medication can have a slowing effect on human gastric emptying [16]. While this technique not only uses irradiation but is also prone to movement artefacts [17], we applied a more robust ${ }^{13} \mathrm{C}$-Sodium OBT paradigm to replicate and quantify this important iatrogenic effect in healthy aged controls, as dopaminergic medication remains the mainstay of treatment for PD.

\section{Methods}

This prospective cross-sectional trial (clinicaltrials.gov Identifier NCT01518751) was approved by the local ethics committee. Written informed consent was obtained from all participants prior to participation. Sixteen Hoehn \& Yahr (H\&Y) stage 2 idiopathic PD patients (PD) according to UKPD brain bank criteria, and 11 age- and sex-matched controls (Ctrl1) were recruited from our movement disorders outpatient clinic and the general population. We did not keep a screening log. 10 healthy young male controls (Ctrl2) were included from internal laboratory data (Table 1). None of the participants were active smokers. The effect of Levodopa on GE was tested in 7 additional healthy subjects (Crtrl3). Subjects with medication influencing blood pressure or gastrointestinal motility (including anticholinergics, antidepressants, neuroleptics, laxatives and prokinetic drugs), significant systemic illness (such as liver/kidney failure, thyroid disease, autoimmune disorders etc.), hormone replacement therapy, BMI $<18$ or $>32 \mathrm{~kg} / \mathrm{m}^{2}$, symptoms or past medical history of GI disease or surgery and diabetes mellitus were excluded from participation.

Patients were on stable dopaminergic treatment prior to inclusion and stopped dopamine agonists, MAO-B-inhibitors and slow-release Levodopa formulations $36 \mathrm{~h}$,

Table 1 Demographic and clinical characteristics of study participants: clinical data of Parkinson disease patients (PD, $n=16)$, age-and sexmatched controls (Ctrl1, $n=11)$ and young male controls $(\mathrm{Ctrl} 2, n=10)$; values given in mean $\pm \mathrm{SD}$

\begin{tabular}{lllll}
\hline & PD $(n=16)$ & Ctrl1 $(n=11)$ & Ctrl2 $(n=10)$ & Differences between groups \\
\hline Participant characteristics & & & \\
Age (years) & $66.3 \pm 8.5$ & $61.1 \pm 7.1$ & $29.7 \pm 6.1$ & $<0.001^{*} ;<0.001^{* ;} 0.26^{\# ;}<0.001 \%$ \\
Sex (male/female) & $10 / 6$ & $7 / 4$ & $10 / 0$ & $\chi^{2}=0.08$ \\
BMI $\left(\mathrm{kg} / \mathrm{m}^{2}\right)$ & $25.4 \pm 3.2$ & $24.1 \pm 4.2$ & $24.4 \pm 1.3$ & $0.53^{*}$ \\
MoCA & $25.9 \pm 2.1$ & $26.9 \pm 2.1$ & n.a. & $0.25^{\dagger}$ \\
UPDRS III (in OFF state) & $28.4 \pm 10.4$ & $1.1 \pm 1.1$ & n.a. & $\leq 0.001^{\dagger}$ \\
Subjective gastrointestinal symptoms & & & $0.22^{*}$ \\
GCSI & $2.5 \pm 3.1$ & $1.8 \pm 4.5$ & $0.2 \pm 0.3$ & $0.60^{*}$ \\
LDQ & $1.5 \pm 1.5$ & $0.9 \pm 1.2$ & $1.1 \pm 2.0$ & \\
\hline
\end{tabular}

$\chi^{2}$ Chi square-test

BMI body mass index, MoCA Montreal cognitive assessment, UPDRS United Parkinsons Disease Rating Scale, GCSI Gastroparesis Cardinal Symptom Index, LDQ leeds dysphagia questionnaire

* $p$ values ANOVA test across all three groups

* $p$ value Bonferroni post hoc comparison between groups PD vs. Ctrl2

\# $p$ value Bonferroni post hoc comparison between groups PD vs. Ctrl1

$\% p$ value Bonferroni post hoc comparison between groups Ctrl1 vs. Ctrl2

${ }^{\dagger} p$ value Student's $t$ test comparing PD vs. Ctrl1 
Levodopa and catechol-O-methyltransferase (COMT)-inhibitors $12 \mathrm{~h}$ before investigations.

\section{GE and visceral perception in PD}

The previously described ${ }^{13} \mathrm{C}$-Sodium OBT [8] consisted of a solid test meal in the form of two muffins $(428 \mathrm{kcal}$, $12.5 \mathrm{~g}$ protein, $18 \mathrm{~g}$ fat, $53 \mathrm{~g}$ carbohydrates) labelled with $125 \mathrm{mg}$ of ${ }^{13} \mathrm{C}$-Sodium Octanoate (isotopic purity of $99.1 \%$, Euriso-Top, Gif-sur-Yvette, France) and $100 \mathrm{ml}$ of tap water: in short, ingested ${ }^{13} \mathrm{C}$ sodium octanoate is emptied from the stomach, absorbed in the intestine and then metabolised to be ultimately exhaled as ${ }^{13} \mathrm{CO}_{2}$. The rate limiting step for this is the velocity of gastric emptying $[8,17]$. The ${ }^{13} \mathrm{CO}_{2} /{ }^{12} \mathrm{CO}_{2}$ isotopic ratio in breath samples was determined with isotope-selective spectrometry (Kibion AB, Uppsala, Sweden) and expressed as the exhaled percentage (of total ingested ${ }^{13} \mathrm{C}$ ) dose rate per hour (PDR in \%/h; Fig. 1a). After an overnight fast, all subjects were tested at 8:00-10:00 AM in the same examination room at ambient temperature. A VAS for subjective appetite intensity, nausea, bloating and abdominal pain was to be scored by the subjects in parallel to the ongoing breath sampling at each time point. VAS data was analysed by quantification of the area under the curve (AUC) by the trapezoid method. After a 20 min supine rest and baseline measurements all subjects ingested the complete test meal. Breath samples and VAS recordings were collected in 15 min-intervals for $4 \mathrm{~h}$. During testing, subjects remained in a relaxed $40^{\circ}$ upright position and physical activity was restricted. Gastric emptying parameters were calculated after performing a non-linear regression of the PDR curve as described previously [8]. Gastric half emptying time (t50) and the emptying coefficient (GEC) were calculated from raw data, the latter being a global index of gastric emptying representing the natural logarithm of the initial slope of the PDR curve $[8,18]$.

\section{Effect of levodopa on GE in healthy aged volunteers}

In a separate experimental setup, seven aged, healthy, nonsmoking controls (4 males, 48-70 years, no previous medical history, no regular medication) received a solid test meal muffin $(241 \mathrm{kcal}, 14 \mathrm{~g}$ proteins, $26 \mathrm{~g}$ carbohydrates, $9 \mathrm{~g}$ fat) labelled with $100 \mathrm{mg}{ }^{13} \mathrm{C}$-Sodium Octanoate. After baseline measurements and immediately before test meal ingestion, subjects received either placebo or $100 \mathrm{mg}$ levodopa $/ 25 \mathrm{mg}$ benserazide on two separate days after an overnight fast. Medication/placebo was administered in a single-blinded manner together with $10 \mathrm{ml}$ of water. All other experimental conditions of the ${ }^{13} \mathrm{C}$-Sodium OBT paradigm and analysis were performed as described above.

\section{Statistical analysis}

We performed Student's $t$ test or one-way ANOVA with Bonferroni-post hoc analysis for comparison of means, and Pearson's Chi Square test for comparison of nominal data. Comparison of variance was performed with the Levene test. Correlation was expressed by Pearson's coefficient. A $p$-level of $<0.05$ was regarded as significant. Analyses were performed using GraphPad Prism 6.0 (GraphPad Software Inc., CA, USA) and IBM(R) SPSS(R) 22 (IBM Corporation, NY, USA).

\section{Results}

\section{Demographics and gastrointestinal symptoms}

Participating PD and Ctrl1 subjects did not differ with regards to sex and age, while male Ctrl2 subjects were significantly younger (Table 1). PD, Ctrl1 and Ctrl2 subjects were matched for symptoms of dyspepsia and gastroparesis by validated standardized questionnaires (Leeds Dysphagia Questionnaire-LDQ [19], Gastroparesis Cardinal Symptom Index-GCSI [20]; one-way ANOVA $p>0.05$ ) and body mass index (BMI; one-way ANOVA $p=0.53$; see Table 1). In the cohort studied, on average, PD patientś age was $66.3 \pm 8.5$ years at the time of study and mean disease duration $5.5 \pm 4.5$ years. Mean Levodopa-equivalent daily dose (LED), calculated from all dopaminergic medication [21], was $694 \pm 340 \mathrm{mg}$ : patients were treated with dopaminergic medication and a MAO inhibitor $(n=7)$, dopamine agonists only $(n=3)$, levodopa only $(n=3)$, or a combination of both. No patients after deep brain stimulation were included. Mean UPDRS III in the OFF state was $28.4 \pm 10.4$. According to the most prominent motor features upon clinical examination, disease phenotype was grouped as tremor-dominant $(6 / 16)$, akinetic-rigid (7/16) or mixed (3/16).

\section{Gastric emptying and pre-/postprandial visceral perception}

Gastric half emptying time T50 (min) did not differ between PD $(215.18 \pm 96.88)$ and both Ctrl1 $(196.16 \pm 53.01)$ and $\mathrm{Ctrl} 2$ subjects $(216.28 \pm 32.81$; oneway ANOVA $p=0.76$, see Fig. 1). GEC did not differ between PD (3.07 \pm 0.39$)$, and both Ctrl1 $(3.05 \pm 0.39)$ and Ctrl2 subjects $(2.77 \pm 0.19$; one-way ANOVA $p=0.09$, Fig. 1c, d). T50 also did not differ between tremor-dominant $\quad(206.21 \pm 36.95, \quad n=6), \quad$ mixed $(167.78 \pm 34.75, n=3)$ and akinetic-rigid PD subjects $(243.18 \pm 140.74, n=7$; one-way ANOVA $p=0.65)$. One Ctrl1 (9\%) and 2 akinetic-rigid PD patients (13\%) 
A

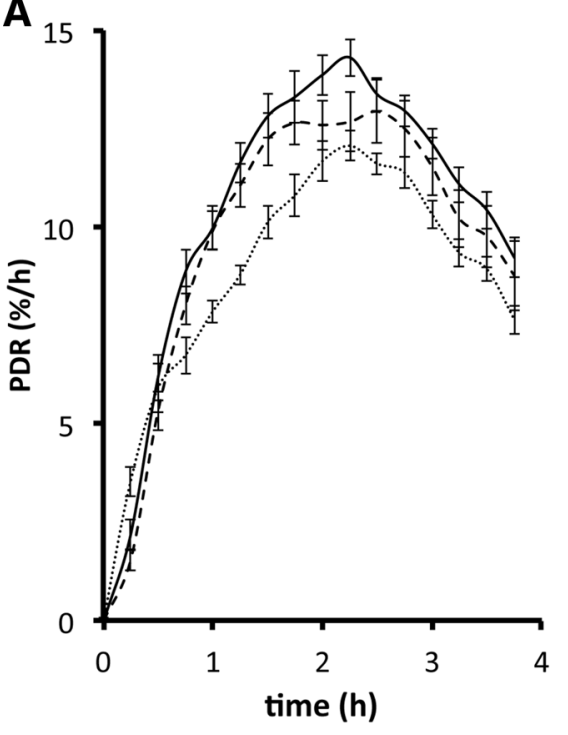

C

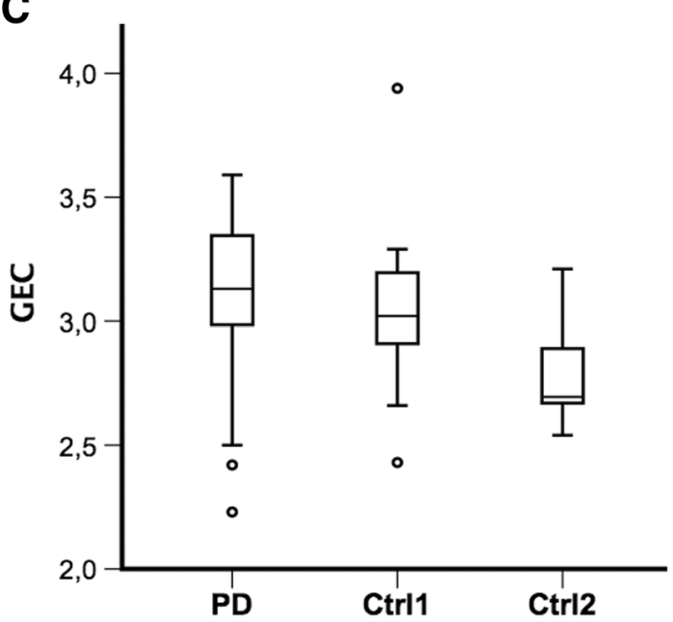

E

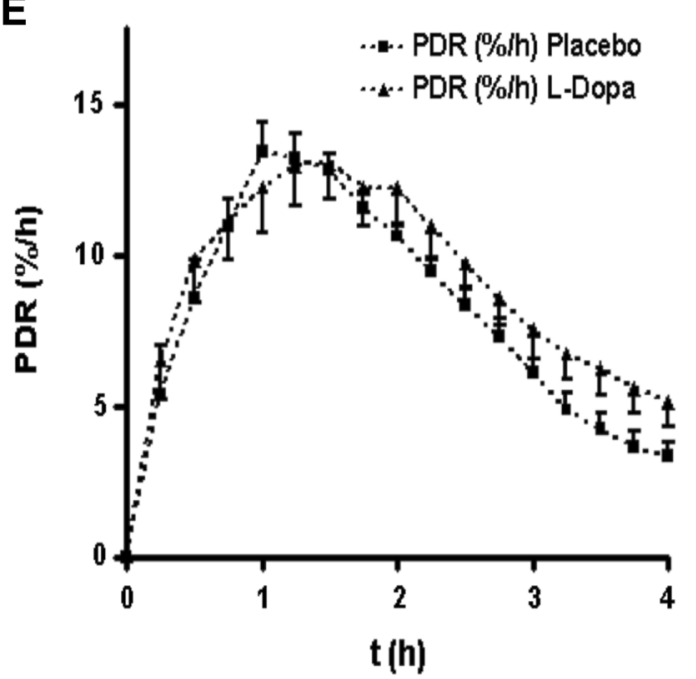

B
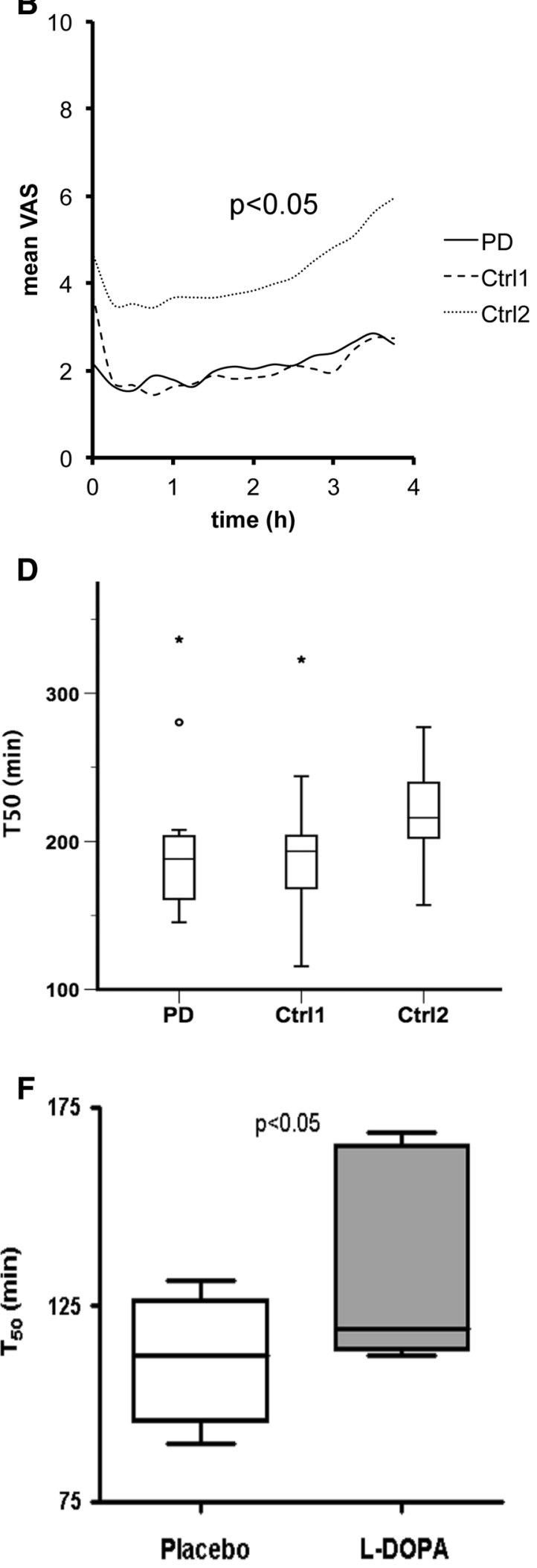
4Fig. 1 Quantification of gastric emptying and subjective visceral perception: data from PD patients (PD), sex- and age-matched (Ctrl1) and young male controls (Ctrl2) were compared. Gastric emptying kinetics as ${ }^{13} \mathrm{CO}_{2} /{ }^{12} \mathrm{CO}_{2}$ isotopic ratio in breath samples were determined with isotope-selective spectrometry and expressed as the exhaled percentage (of total ingested ${ }^{13} \mathrm{C}$ ) dose rate per hour (PDR in $\% / \mathrm{h}$; a); Mean appetite sensation was measured by visual analogue scales in parallel to the ongoing breath sampling over $4 \mathrm{~h}$ and the area under the curve quantified by the trapezoid method (b); Gastric emptying coefficient (GEC; c) and gastric emptying half time $\left(T_{50}\right.$; d) were calculated from gastric emptying raw data. In a separate experiment gastric emptying in healthy aged controls $(n=7)$ was examined after oral placebo or $100 \mathrm{mg}$ Levodopa, resulting in a significant change in gastric emptying kinetics (e) and t50 (f)

had gastroparesis, defined by t50 > $300 \mathrm{~min}$. Comparison of variance in between motor phenotype PD patient groups showed a significantly higher variance in T50 values in the akinetic-rigid group in comparison to tremor-dominant and mixed phenotype (Levene test $F=4.27, p=0.04$ ). Patients of different motor phenotype did not differ with regards to LED, LED/kg body weight or number of dopaminergic medication taken.

Quantification of VAS data for appetite differed significantly between groups and was higher in Ctrl2 $(1031 \pm 351)$ than PD or Ctrl1 subjects $(513 \pm 447$; $496 \pm 583$; one-way ANOVA $p=0.02$; Fig. $1 b$ ), while there was no difference on nausea $(21.6 \pm 31.2$; $18.5 \pm 23.7 ; \quad 3.9 \pm 11.3 ; \quad p=$ n.s.), bloating (97.9 \pm $224.4 ; 24.3 \pm 31.2 ; 71.5 \pm 125.1 ; p=$ n.s.), and abdominal pain $(22.2 \pm 36.5 ; \quad 17.5 \pm 21.4 ; \quad 85.5 \pm 228.4$; $p=$ n.s.) between groups. GEC/t50 correlated neither with age, BMI or sex, nor with disease duration, motor impairment (UPDRS III score) or LED in PD patients.

\section{Influence of Levodopa on gastric emptying in healthy aged subjects}

In comparison to placebo, oral ingestion of $100 \mathrm{mg}$ Levodopa/25 mg Benserazide caused a significant slowing of GE in healthy elderly control subjects (t50 (min): $112 \pm 15$ vs. $132 \pm 24 p=0.04$; GEC: $3.67 \pm 0.25$ vs. $3.34 \pm 0.31 p=0.04$; Fig. 1e, f).

\section{Discussion}

In this cross-sectional, prospective study using a high caloric test meal in the strict absence of dopaminergic medication, there was no overall delayed GE in a homogenous, representative sample of early motor PD patients in comparison with healthy, age-matched and younger controls. Neither PD patient motor impairment as measured by UPDRS III scores, disease duration nor LED correlated with GE measures.
Although the comparison of different motor phenotype showed a higher inter-individual variance in GE and gastroparesis $(n=2)$, only in akinetic-rigid patients, our study is not powered to imply a role of PD motor phenotype on GE.

\section{Comparison with previous PD GE studies}

These findings contrast with earlier reports of delayed GE in PD patients $[6-12,18]$, warranting careful interpretation. Dopaminergic medication has a marked influence on GE as shown previously [22] and in this study (Fig. 1e, f). It is therefore difficult to exclude potential medication bias in studies that performed GE measurements not in the dopaminergic OFF condition [6, 7]. The results from study cohorts not matched for age [6] or consisting of advanced disease stage PD patients only [10] are difficult to compare with our results because of substantial methodological differences. Furthermore, it is has been shown that GE studied by liquid test meals more often gives abnormal results [23] and shows a larger variability than solid test meals [24], rendering PD studies using liquid test meals difficult to interpret [12].

Previous studies in early motor-stage PD patients that used a solid test meal off dopaminergic medication found delayed GE $[8,9,11]$. Patients in the Caucasian cohorts did not differ from our sample with respect to age, gender distribution (male preponderance), disease onset, disease duration and BMI-they received dopaminergic medication but LED was not calculated [8, 11]. In the Japanese study, patients were drug-naïve, had a shorter disease duration, a different gender distribution (female preponderance) and a lower BMI [9]. Identical to our paradigm, these studies applied a ${ }^{13} \mathrm{C}$-Sodium Octanoate breath test after an overnight fast in a similar manner. However, they used a $240 \mathrm{kcal}$ caloric test meal (calorically corresponding to one butter croissant), which is only about half the caloric content of our paradigm. Dopaminergic medication was stopped $12 \mathrm{~h}$ before testing $[8,11]$ /excluded long-acting dopaminergic medication [8], whereas in our study, all medication potentially affecting levodopa metabolism was stopped $36 \mathrm{~h}$ and short-acting dopamine $12 \mathrm{~h}$ before testing. In our opinion, there are several aspects that might explain the rather counterintuitive finding of unchanged GE in our sample:

\section{Influence of humoral factors on GE}

It is known that there is a positive linear correlation between caloric intake and GE time of solid meals, which has been demonstrated using the ${ }^{13} \mathrm{C}$-Sodium Octanoate breath test and other methods [24-26]. Physiologically, GE is regulated by both the influence of local vago-vagal 
reflexes and the release of GI hormones [27]. The humoral response during GE consists of prokinetic (Ghrelin) and inhibitory factors [glucagon-like peptide 1 (GLP-1), pancreatic polypeptide Y (PPY)]. Physiologically, a postprandial increase of GLP-I and a significant drop in Ghrelin prevents an overshoot of GE and fine tunes the release of nutrients from the stomach into the gut [28]. There is data to suggest that this humoral response regulating gastric motility after food intake is altered in early PD patients: after the administration of a $214 \mathrm{kcal}$ test meal, serum Ghrelin levels have been found to recuperate less after a physiological initial drop in PD patients than in controls, while serum GLP-1 levels were unchanged. This decreased prokinetic and unchanged inhibitory signaling is in concordance with the observed slowing of GE among PD patients $[29,30]$.

We speculate that the caloric content of the test meal might play a role in explaining the apparent discrepancies outlined above. The extent of the humoral GI response depends on the caloric content of the meal [31] and is regulated, among others, by ileal brake mechanisms [32, 33]. Although we did not measure GI hormone release, we speculate that the higher caloric content of our test meal might have triggered a higher Ghrelin response, potentially influencing GE by a humoral response quantitatively different from the response after a smaller test meal.

We acknowledge the finding of delayed GE in untreated, early motor stage PD patients after a low caloric test meal $[9,11]$. Nevertheless, a high caloric test meal more adequately mimics the nutritional intake in the majority of PD patients with respect to caloric and nutritional content. Therefore, this paradigm more adequately addresses the question whether there is a diseaseinherent slowing of GE in early motor stage PD. Our results show that $\mathrm{H} \& \mathrm{Y} 2 \mathrm{PD}$ patients overall do not have clinically relevant delayed GE of a medium sized meal in the absence of medication.

\section{Influence of the vagal nerve on GE}

The autonomic function of the enteric nervous system is modulated by vagal nerve efferents. Alternatively to an altered humoral response pattern, GE might be altered by a changed gastric vagal motor control, as previously suggested $[8,9,34,35]$. There is histopathological evidence suggesting a connection between alpha-synuclein immunoreactive inclusions in the gastric myenteric and submucosal plexus and the brainstem through the vagal nerve in PD [36]. However, in contrast to the neurodegeneration caused in central structures in PD, this alphasynuclein accumulation does not cause neurodegeneration in the enteric nervous system, i.e. does not change neuron numbers [37]. Furthermore, while local vago-vagal reflexes seem to mainly affect gastric distribution of a meal during the non-propulsive Lag-phase and sensation of fullness [27], GE less clearly depends on vagal integrity as it remains unchanged after vagotomy [38]. Due to the nature of autonomic function testing, so far a functional impairment of vagal nerve activity in PD patients has not been proven, although hypothesized.

Our results of unchanged appetite perception indicate that afferent vagal nerve functioning is not impaired in early motor PD patients. Physiologically, satiety is induced by gastric distension and mediated via vagal afferents to CNS structures [27]. The feeling of satiety is created by interplay of numerous hormonal and neuronal signals both on a peripheral and central level, that ultimately contribute to the regulation of food intake [39]. While it is known that food intake in PD can be altered on various levels from afferent metabolic stimuli, sensory perception to mood and motivation [40], subjective appetite perception relies on satiety signalling, i.e. vagal afferent functioning. Concluding from our results, this feedback mechanism is not impaired (sufficiently) to affect appetite perception in early-motor PD patients.

\section{Influence of dopaminergic medication on GE}

The finding of a significant slowing of GE after Levodopa intake in healthy aged subjects corroborates results of gamma-scintigraphy experiments $[16,22,41]$ and replicates them for the first time using the ${ }^{13} \mathrm{C}$-Sodium OBT paradigm. Interestingly, already our small sample was sufficient to show a significant $18 \%$ slowing of GE after $100 \mathrm{mg}$ oral Levodopa-together with the significant slowing in equally small sample sizes reported by Robertson et al. $(n=8)$ and Berkowitz et al. $(n=7)$, indicating a robust effect of dopamine on gastric motility $[16,22,41]$. Due to the single dose paradigm used in our study, we cannot deduct a dosedependent slowing effect of dopamine on GE, although animal data does suggest this [42]. Furthermore, although an animal study found Ghrelin levels not to be significantly influenced by intravenous levodopa [43], it is not known in how far dopaminergic medication per se influences GE humoral response mechanisms in humans.

The acute effect of levodopa on gastric motility in PD subjects has not been investigated so far. Our results not only stress the importance of performing GE examinations in the strict absence of dopaminergic agents when assessing disease-intrinsic GI motility, but also document a marked iatrogenic effect of oral dopaminergic therapy. They do suggest that at least part of the clinically relevant symptoms of delayed GE in H\&Y, 2 PD patients might be related to treatment rather than disease-inherent effects. Future studies should address this effect of levodopa on GE in different disease stages. 
Gastric motility is of central importance to the pharmacokinetics in PD since Levodopa, still the mainstay of oral dopaminergic treatment, is absorbed only in the small intestine $[44,45]$. Due to peripheral decarboxylation of Levodopa to dopamine in the gut mucosa and liver, the bioavailability of Levodopa with normal GI motility is only about $30 \%$-with delayed propagation, absorption and therefore, more extensive decarboxylation; this number is reduced even further. It has been shown that Levodopa pharmacokinetics and gastric emptying kinetics in PD subjects correlate to a certain extent $[46,47]$. From this, it has been argued that delayed GE causes lower Levodopa plasma levels impacting on the motor response of medication [46, 47]. Our data expand this argument and support the notion that at least in the early-motor phase of the disease, Levodopa to a great extent might influence its own absorption due to its slowing effect on GE.

\section{Appetite perception is unchanged in early motor PD}

Visceral perception in PD has barely been studied so far, although unintended weight loss has a high prevalence in advanced disease stages [13] and is associated with a less favourable outcome [14]. Our results indicate an age- but no disease-related effect in appetite perception in early motor PD and therefore are less likely to account for unintended weight loss. This is in line with the finding of decreased appetite sensation with higher age, the so-called anorexia of aging [48]. For later PD disease stages, subjective appetite perception remains to be studied.

\section{Limitations and conclusion}

We are aware of the limitations of this study. Above all, the relatively small sample size increases the risk for type II error and warrants a substantiation of the preliminary results of the sub-group analysis with regards to the influence of motor phenotype on non-motor symptoms. We also acknowledge that serial measurements of GI hormones, especially intestinal satiety signals such as cholecystokinin, GLP-1, Ghrelin and PPY after test meals of different caloric size would further substantiate the discussion of our results. Finally, the effect of levodopa on gastric motility in different disease stages of PD and its dose-dependency should be addressed in future studies.

In summary, this study did not find an overall slowing of GE in early-motor PD using a high caloric test meal. Delayed GE is an important but neglected iatrogenic side effect of L-Dopa treatment that needs to be taken into account in the treatment of individual patients. We conclude that clinically relevant GE slowing in early PD is, however, caused by the iatrogenic effect of dopamine treatment. Visceral perception for appetite shows an age- but no disease-related effect in early motor-stage PD, which indicates largely intact vagal afferent signalling in this disease stage.

Acknowledgments SRS is supported by study grants by Parkinson Schweiz and the EMDO foundation, Zurich, Switzerland.

Conflicts of interest LE, OG, DiW, CB and DaW report no financial disclosures.

\section{References}

1. Adler CH (2005) Nonmotor complications in Parkinson's disease. Mov Disord 1(20 Suppl 11):S23-S29

2. Gallagher DA, Lees AJ, Schrag A (2010) What are the most important nonmotor symptoms in patients with Parkinson's disease and are we missing them? Mov Disord 25(15):2493-2500

3. Verbaan D, Marinus J, van Rooden SM, Stiggelbout AM, van Hilten JJ, Visser M (2007) Patient-reported autonomic symptoms in Parkinson disease. Neurology. 69(4):333-341

4. Antonini A, Barone P, Marconi R, Morgante L, Zappulla S, Pontieri FE et al (2012) The progression of non-motor symptoms in Parkinson's disease and their contribution to motor disability and quality of life. J Neurol 259(12):2621-2631

5. Pfeiffer RF (2003) Gastrointestinal dysfunction in Parkinson's disease. Lancet Neurol 2(2):107-116

6. Djaldetti R, Baron J, Ziv I, Melamed E (1996) Gastric emptying in Parkinson's disease: patients with and without response fluctuations. Neurology 46(4):1051-1054

7. Hardoff R, Sula M, Tamir A, Soil A, Front A, Badarna S et al (2001) Gastric emptying time and gastric motility in patients with Parkinson's disease. Mov Disord 16(6):1041-1047

8. Goetze O, Wieczorek J, Mueller T, Przuntek H, Schmidt WE, Woitalla D (2005) Impaired gastric emptying of a solid test meal in patients with Parkinson's disease using 13C-sodium octanoate breath test. Neurosci Lett 375(3):170-173

9. Tanaka Y, Kato T, Nishida H, Yamada M, Koumura A, Sakurai T et al (2010) Is there a delayed gastric emptying of patients with early-stage, untreated Parkinson's disease? An analysis using the 13C-acetate breath test. J Neurol 258(3):421-426

10. Tanaka Y, Kato T, Nishida H, Araki H, Murase M, Nagaki M et al (2009) Is there a difference in gastric emptying between Parkinson's disease patients under long-term 1-dopa therapy with and without motor fluctuations? An analysis using the 13C-acetate breath test. J Neurol 256(12):1972-1976

11. Unger MM, Moeller JC, Mankel K, Schmittinger K, Eggert KM, Stamelou M et al (2011) Patients with idiopathic rapid-eyemovement sleep behavior disorder show normal gastric motility assessed by the 13C-octanoate breath test. Mov Disord 26(14):2559-2563

12. Thomaides T, Karapanayiotides T, Zoukos Y, Haeropoulos C, Kerezoudi E, Demacopoulos N et al (2005) Gastric emptying after semi-solid food in multiple system atrophy and Parkinson disease. J Neurol 252(9):1055-1059

13. Abbott RA, Cox M, Markus H, Tomkins A (1992) Diet, body size and micronutrient status in Parkinson's disease. Eur J Clin Nutr 46(12):879-884

14. Sharma JC, Vassallo M (2014) Prognostic significance of weight changes in Parkinson's disease: the Park-weight phenotype. Neurodegener Dis Manag. 4(4):309-316

15. Sheard JM, Ash S, Mellick GD, Silburn PA, Kerr GK (2013) Malnutrition in a sample of community-dwelling people with Parkinson's disease. PLoS One 8(1):e53290 
16. Robertson DR, Renwick AG, Wood ND, Cross N, Macklin BS, Fleming JS et al (1990) The influence of levodopa on gastric emptying in man. Br J Clin Pharmacol 29(1):47-53

17. Ghoos YF, Maes BD, Geypens BJ, Mys G, Hiele MI, Rutgeerts PJ et al (1993) Measurement of gastric emptying rate of solids by means of a carbon-labeled octanoic acid breath test. Gastroenterology 104(6):1640-1647

18. Goetze O, Nikodem AB, Wiezcorek J, Banasch M, Przuntek H, Mueller T et al (2006) Predictors of gastric emptying in Parkinson's disease. Neurogastroenterol Motil 18(5):369-375

19. Fraser A, Delaney B, Ford A, Qume M, Moayyedi P (2007) The short-form leeds dyspepsia questionnaire validation study. Aliment Pharmacol Ther 25(4):477-486

20. Revicki DA, Rentz AM, Dubois D, Kahrilas P, Stanghellini V, Talley NJ et al (2003) Development and validation of a patientassessed gastroparesis symptom severity measure: the Gastroparesis Cardinal Symptom Index. Aliment Pharmacol Ther 18(1):141-150

21. Tomlinson CL, Stowe R, Patel S, Rick C, Gray R, Clarke CE (2010) Systematic review of levodopa dose equivalency reporting in Parkinson's disease. Mov Disord 25(15):2649-2653

22. Robertson DR, Renwick AG, Macklin B, Jones S, Waller DG, George CF et al (1992) The influence of levodopa on gastric emptying in healthy elderly volunteers. Eur J Clin Pharmacol 42(4):409-412

23. Ziessman HA, Okolo PI, Mullin GE, Chander A (2009) Liquid gastric emptying is often abnormal when solid emptying is normal. J Clin Gastroenterol 43(7):639-643

24. Maes BD, Ghoos YF, Geypens BJ, Hiele MI, Rutgeerts PJ (1995) Relation between gastric emptying rate and energy intake in children compared with adults. Gut 36(2):183-188

25. Calbet JA, MacLean DA (1997) Role of caloric content on gastric emptying in humans. J Physiol 498(Pt 2):553-559

26. Peracchi M, Gebbia C, Ogliari C, Fraquelli M, Viganò R, Baldassarri A et al (2000) Influence of caloric intake on gastric emptying of solids assessed by $13 \mathrm{C}$-octanoic acid breath test. Scand J Gastroenterol 35(8):814-818

27. Janssen $P$, Vanden Berghe $P$, Verschueren S, Lehmann A, Depoortere I, Tack J (2011) Review article: the role of gastric motility in the control of food intake. Aliment Pharmacol Ther 33(8):880-894

28. Hellström PM, Grybäck P, Jacobsson H (2006) The physiology of gastric emptying. Best Pract Res Clin Anaesthesiol 20(3):397-407

29. Unger MM, Möller JC, Mankel K, Eggert KM, Bohne K, Bodden $M$ et al (2011) Postprandial ghrelin response is reduced in patients with Parkinson"s disease and idiopathic REM sleep behaviour disorder: a peripheral biomarker for early Parkinson"s disease? J Neurol 258(6):982-990

30. Unger MM, Ekman R, Björklund A-K, Karlsson G, Andersson C, Mankel K et al (2013) Unimpaired postprandial pancreatic polypeptide secretion in Parkinson's disease and REM sleep behavior disorder. Mov Disord 28(4):529-533

31. Rijkelijkhuizen JM, McQuarrie K, Girman CJ, Stein PP, Mari A, Holst JJ et al (2010) Effects of meal size and composition on incretin, alpha-cell, and beta-cell responses. Metab Clin Exp 59(4):502-511

32. Vollmer K, Gardiwal H, Menge BA, Goetze O, Deacon CF, Schmidt WE et al (2009) Hyperglycemia acutely lowers the postprandial excursions of glucagon-like Peptide-1 and gastric inhibitory polypeptide in humans. J Clin Endocrinol Metab 94(4):1379-1385

33. Meier JJ, Gallwitz B, Salmen S, Goetze O, Holst JJ, Schmidt WE et al (2003) Normalization of glucose concentrations and deceleration of gastric emptying after solid meals during intravenous glucagon-like peptide 1 in patients with type 2 diabetes. J Clin Endocrinol Metab 88(6):2719-2725

34. Heetun ZS, Quigley EMM (2012) Gastroparesis and Parkinsońs disease: a systematic review. Parkinsonism Relat Disord. 18(5):433-440

35. Marrinan S, Emmanuel AV, Burn DJ (2013) Delayed gastric emptying in Parkinson's disease. Mov Disord 29(1):23-32

36. Braak H, de Vos RA, Bohl J, Del Tredici K (2006) Gastric alphasynuclein immunoreactive inclusions in Meissner's and Auerbach's plexuses in cases staged for Parkinson's disease-related brain pathology. Neurosci Lett 396(1):67-72

37. Annerino DM, Arshad S, Taylor GM, Adler CH, Beach TG, Greene JG (2012) Parkinson's disease is not associated with gastrointestinal myenteric ganglion neuron loss. Acta Neuropathol 124(5):665-680

38. Sheiner HJ, Quinlan MF, Thompson IJ (1980) Gastric motility and emptying in normal and post-vagotomy subjects. Gut 21(9):753-759

39. Begg DP, Woods SC (2013) The endocrinology of food intake. Nature Rev Endocrinol 9(10):584-597

40. Aiello M, Eleopra R, Rumiati RI (2014) Body weight and food intake in Parkinson's disease. A review of the association to nonmotor symptoms. Appetite 23(84C):204-211

41. Berkowitz DM, McCallum RW (1980) Interaction of levodopa and metoclopramide on gastric emptying. Clin Pharmacol Ther 27(3):414-420

42. Nagahata Y, Azumi Y, Kawakita N, Wada T, Saitoh Y (1995) Inhibitory effect of dopamine on gastric motility in rats. Scand $\mathbf{J}$ Gastroenterol 30(9):880-885

43. Wang L, Murphy NP, Stengel A, Goebel-Stengel M, St Pierre DH, Maidment NT et al (2012) Ghrelin prevents levodopa-induced inhibition of gastric emptying and increases circulating levodopa in fasted rats. Neurogastroenterol Motil 24(5):e235e245

44. Nyholm D, Lennernäs H (2008) Irregular gastrointestinal drug absorption in Parkinson's disease. Expert Opin Drug Metab Toxicol 4(2): 193-203

45. Kurlan R, Rothfield KP, Woodward WR, Nutt JG, Miller C, Lichter D et al (1988) Erratic gastric emptying of levodopa may cause "random" fluctuations of parkinsonian mobility. Neurology 38(3):419-421

46. Doi H, Sakakibara R, Sato M, Masaka T, Kishi M, Tateno A et al (2012) Plasma levodopa peak delay and impaired gastric emptying in Parkinson's disease. J Neurol Sci 319(1-2):86-88

47. Müller T, Erdmann C, Bremen D, Schmidt WE, Muhlack S, Woitalla D et al (2006) Impact of gastric emptying on levodopa pharmacokinetics in Parkinson disease patients. Clin Neuropharmacol 29(2):61-67

48. Moss C, Dhillo WS, Frost G, Hickson M (2012) Gastrointestinal hormones: the regulation of appetite and the anorexia of ageing. J Hum Nutr Diet 25(1):3-15 